\title{
CONCRETOS CONTAMINADOS POR PIRITA: CORROSÃO E FORMAÇÃO DE PELÍCULA PASSIVADORA DO AÇO
}

\author{
CAPRARO, ANA PAULA \\ Professora Dra., Engenheira Civil \\ Universidade Federal do Paraná \\ Paraná; Brasil \\ anapcapraro@gmail.com
}

\author{
CALVO, ANALIET \\ Doutoranda, Engenheira Civil \\ Universidade Federal do Paraná \\ Paraná; Brasil \\ analietcv@yahoo.es
}

\author{
MEDEIROS, MARCELO H. F \\ Professor, Dr., Engenheiro Civil \\ Universidade Federal do Paraná \\ Paraná; Brasil \\ medeiros.ufpr@gmail.com \\ PIERALISI, RICARDO \\ Professor, Dr., Engenheiro Civil \\ Universidade Federal do Paraná \\ Paraná; Brasil \\ ricpieralisi@ufpr.br
}

\begin{abstract}
RESUMO
$\mathrm{O}$ ataque interno de sulfatos pode consumir, entre outros, a portlandita responsável pela manutenção do pH do concreto. Além disso, as variações volumétricas e as fissurações causadas pelo fenômeno podem facilitar a entrada de outros agentes agressivos, favorecendo a corrosão de armaduras. Com o objetivo de estudar a relação, entre a reação sulfática interna (RSI) e a corrosão, foram moldados corpos de prova de concreto armado, de $25 \times 25 \times 7 \mathrm{~cm}$, com três teores diferentes de contaminação por pirita $(0,38 \% ; 0,75 \%$ e $3,75 \%)$, e uma série referência, sem contaminação. As amostras permaneceram em cura submersa até os 28 dias, e, após, foram expostas a uma condição acelerada em um tanque com solução aquosa constantemente aerada. Para a análise do mecanismo de corrosão foram realizadas leituras de densidade de corrente, potencial de circuito aberto e resistividade elétrica do concreto, até os 1100 dias de idade. Através dos resultados obtidos foi possível identificar que as técnicas empregadas puderam diferenciar a série referencia das demais e, ainda, indicar a maior suceptibilidade da série de maior contaminação $(3,75 \%)$ ao mecanismo de corrosão.
\end{abstract}

Palavras-chave: ataque interno de sulfatos, contaminação do agregado, corrosão de armaduras, densidade de corrente, condição acelerada.

\begin{abstract}
The internal sulfate attack may consume the portlandite responsible for maintaining the concrete $\mathrm{pH}$. In addition, the volumetric deformation and cracks caused by this phenomenon may facilitate the entry of other aggressive agents, favoring the corrosion of steel reinforcement. In order to study the relationship between the internal sulfate reaction (ISR) and corrosion, $25 \times 25 \times 7 \mathrm{~cm}$ reinforced concrete specimens were cast with three different levels of pyrite contamination $(0.38 \%, 0.75 \%$ and $3.75 \%)$, and without contamination (reference). After 28 days of submerged cure, the specimens were placed inside a tank with a constantly aerated aqueous solution to emulate an accelerated environment. Measurements of current density, corrosion potential and electrical resistivity were performed during 1100 days in order to analyze the corrosion mechanism. It was possible to verify that the techniques could differentiate the reference specimens from the others. Also, the results indicate that the specimens with a higher level of contamination (3.75\%) presented more susceptibility to the corrosion mechanism.
\end{abstract}

Keywords: internal sulfate attack, aggregate contamination, reinforcement corrosion, corrosion velocity, accelerated condition.

\section{INTRODUÇÃO}

Em grandes obras de concreto armado, como as barragens, a possibilidade de extrair agregados em seu entorno é primordial para a viabilidade do empreendimento, uma vez que longas distâncias exigem altos custos de transporte. Além 
disso, os agregados são considerados recursos não renováveis (Hasan et al., 2017; Hossack e Thomas, 2015) e nesse contexto pode ser inevitavel o uso de matérias-primas localmente disponíveis contendo íons sulfato e outros íons nocivos para produzir o concreto (Zhuo et al., 2019).

De modo geral, agregados contendo sulfatos são considerados inadequados para uso em estruturas de concreto, uma vez que podem causar danos à durabilidade das estruturas (Hasparyk et al., 2003), desencadeando a reação sulfática interna (RSI). Esse mecanismo de ataque envolve várias reações químicas que podem produzir produtos secundários, como a etringita, o gesso e a taumasita (Hasan et al., 2017; Ikumi et al., 2017; Zhuo et al., 2019). A formação desses produtos resulta na expansão do concreto ou argamassa, o que pode causar rachaduras e, em casos extremos, pode levar à desintegração do material (Ikumi et al., 2014; Campos et al., 2016; Campos et al., 2018; Zanqun et al., 2018).

Os produtos secundários são formados a partir da reação dos dois compostos formados pela oxidação da pirita no agregado (sulfato ferroso, $\mathrm{FeSO}_{4}$, e ácido sulfúrico, $\mathrm{H}_{2} \mathrm{SO}_{4}$ ) com os compostos hidratados do cimento, aluminato tricálcico $\left(\mathrm{C}_{3} \mathrm{~A}\right)$, hidrato de silicato de cálcio (CSH) e hidróxido de cálcio $\left(\mathrm{Ca}(\mathrm{OH})_{2}\right)$ (Oliveira, 2011; Chuanbei et al., 2019), possibilitando um ataque interno, que eventualmente leva a rachaduras no concreto (Lman et al., 2012; Blanco et al., 2019).

Como os produtos secundários são expansivos, a tensão resultante fissura o concreto e expõe o aço de reforço, deixandoos suscetível ao processo de corrosão (Parande et al., 2006). O fenômeno causa a perda da integridade estrutural e afeta a durabilidade das estruturas de concreto armado (Okeniyi et al., 2014; Li et al., 2014).

A corrosão da armadura do concreto ainda é um problema crescente nas últimas décadas, sendo o fator mais significativo pela deterioração prematura e redução da vida útil das estruturas de concreto armado (Hansson et al., 2012). A corrosão do aço no interior do concreto, é o resultado do efeito combinado de vários fatores ambientais (Santos, 2014; Hansson et al., 2012). Trata-se de um processo eletroquímico que resulta em uma perda de função do elemento, requerendo a presença simultânea de umidade e oxigênio para para produzir ferrugem. Como a ferrugem ocupa aproximadamente seis vezes o volume do material original, quando o processo ocorre dentro de um concreto (barras de reforço de aço) são geradas tensões de tração que fissuram o concreto (Maia e Alves, 2017).

Apesar de o concreto ser alcalino, o próprio processo de oxidação do agregado pode gerar na região superficial dos grãos um microclima mais ácido devido à liberação dos íons deletérios como o $\mathrm{SO}_{4}^{-2}$ e o $\mathrm{H}^{+}$(Araujo, 2008; Gomides, 2009), favorecendo o processo de corrosão do aço. Este fato foi ratificado por Casanova et al. (1996), que afirmaram que, apesar do caráter alcalino do concreto, o ambiente circundante imediato frente à reação tendeu a ser significativamente mais ácido devido à liberação de íons de hidrogênio durante a oxidação.

Partindo do princípio de que o ataque interno de sulfatos pode consumir a portlandita, um dos produtos responsáveis pela manutenção do $\mathrm{pH}$ do concreto, e que as variações volumétricas e as fissurações causadas pelo fenômeno possam vir a facilitar a entrada de outros agentes agressivos (Capraro et al., 2017), optou-se pelo estudo do ataque aliado ao mecanismo de corrosão.

Desse modo, este trabalho apresenta resultados e análises de medidas de densidade de corrente, potencial de circuito aberto, e resistividade elétrica do concreto, realizadas em amostras de concreto armado, com diferentes teores de contaminação por pirita $\left(\mathrm{FeS}_{2}\right)$, sujeitas a um mecanismo de aceleração do processo, exposição em um tanque com solução aquosa constantemente aerada. 


\section{MATERIAIS E MÉTODOS}

A análise da influência da contaminação por sulfatos no mecanismo de corrosão foi realizada em amostras prismáticas de concreto, com dimensões de $(25 \times 25 \times 7) \mathrm{cm}$, contendo duas barras de aço CA50 de diâmetro de $8 \mathrm{~mm}$ e $35 \mathrm{~cm}$ de comprimento, como apresentado na Fig. 1.
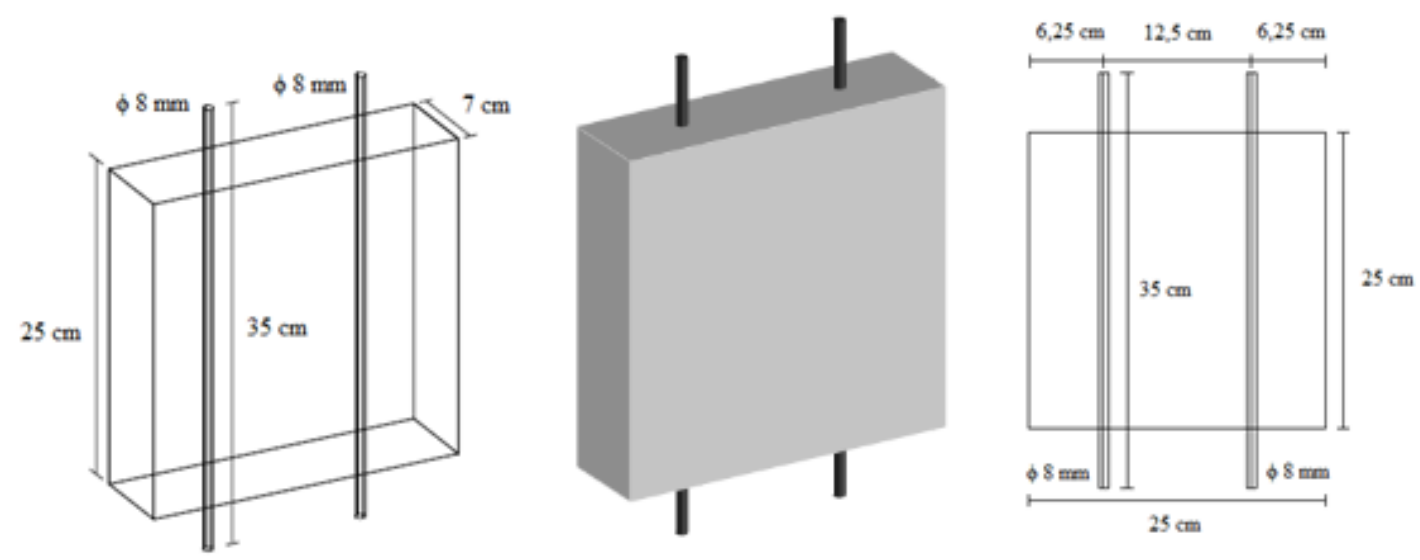

Figura 1: Corpo de prova prismático de concreto armado.

\subsection{Materiais}

O aglomerante empregado nas misturas de concreto foi composto em laboratório, substituindo-se 35\% do CP V - ARI por cinza volante, de modo a aproximar esta mistura de aglomerantes ao cimento tipo CP IV de acordo com a NBR 16697: 2018. Foi feita a escolha do cimento CP IV por este ser muito utilizado em barragens de concreto. Não foi usado diretamente um cimento CP IV comercial porque, neste caso, não se tem a informação do teor exato de pozolana na mistura.

O cimento CP V - ARI empregado possui massa específica de $3,13 \mathrm{~g} / \mathrm{cm}^{3}$ e área específica BET de $1,07 \mathrm{~m}^{2} / \mathrm{g}$. Por outro lado, a cinza volante, proveniente da combustão do carvão mineral da jazida do vale do rio Tubarão, apresenta massa específica de $1,95 \mathrm{~g} / \mathrm{cm}^{3}$ e área específica BET de $1,09 \mathrm{~m}^{2} / \mathrm{g}$.

O agregado miúdo empregado foi uma areia natural, com massa específica de 2,38 g/cm³ (NBR NM 52: 2009), proveniente da região de São Luiz do Purunã, no Paraná. O agregado graúdo foi uma brita 1, com massa específica de $2,72 \mathrm{~g} / \mathrm{cm}^{3}$ (NBR NM 53 : 2009), proveniente da região de Curitiba, Paraná. Nenhum dos agregados empregados possui composição natural com íon sulfeto. Dessa forma, optou-se pela contaminação artificial do agregado miúdo, substituindo parte de sua massa por pirita.

A pirita, contaminante empregada, é proveniente da região de Figueira, no Paraná. Esse material foi obtido de um resíduo do processo de beneficiamento do carvão mineral. Nos ensaios de caracterização o material apresentou massa específica igual a 4,95 g/cm³ e, após o seu processo de moagem, foi classificada como um material passante pela peneira 2,4 mm.

Para contaminação dos materiais, foram considerados quatro níveis de $\mathrm{SO}_{3}$ : sendo um deles de referência sem contaminação $\left(0 \%\right.$ de $\left.\mathrm{SO}_{3}\right)$; dois que segue as recomendações normativas que estabelecem teores máximos de $\mathrm{SO}_{3}$ que podem ser empregados em estruturas de concreto, a saber: $0,5 \%$ e $1,0 \%$; e um acima do recomendado pelas normas (5,0 \%) para avaliação de um teor crítico de contaminação. Neste estudo, o material utilizado foi pirita $\left(\mathrm{FeS}_{2}\right)$, e assim, a concentração de $\mathrm{SO}_{3}$ equivalente foi obtida através do cálculo estequiométrico.

\subsection{Moldagem}

- Estudo concreto:

O traço de concreto adotado foi: 1,0:2,7:2,7 e relação água/cimento de 0,6, decorrente da aplicação do traço na execução de concreto convencional em uma usina hidrelétrica nacional. 
Nesta pesquisa, o material contaminante utilizado foi a pirita $\left(\mathrm{FeS}_{2}\right)$ e, desta forma, para comparação dos teores propostos em $\mathrm{SO}_{3}$ foi realizado o cálculo do teor de enxofre equivalente entre os dois compostos. Na Tabela 1 estão apresentadas as quatro séries de ensaio deste estudo, além de seus respectivos teores de contaminação em $\mathrm{SO}_{3}$ e em pirita com relação à massa de agregados. As taxas de contaminação foram aplicadas sobre a massa total de agregados $(2,7 \mathrm{~kg}$ de agregado miúdo - 2,7 kg de agregado graúdo).

Tabela 1. Teores de contaminação proporcionais em $\mathrm{SO}_{3}$ e pirita.

\begin{tabular}{|c|c|c|}
\hline Série & Teor de $\mathrm{SO}_{3}$ & Teor de pirita $\left(\mathrm{FeS} \mathrm{S}_{2}\right)$ \\
\hline Referência $(0,0 \%)$ & $0,00 \%$ & $0,0 \times 0,75=0,00 \%$ \\
\hline Contaminação $(0,5 \%)$ & $0,50 \%$ & $0,5 \times 0,75=0,38 \%$ \\
\hline Contaminação $(1,0 \%)$ & $1,00 \%$ & $1,0 \times 0,75=0,75 \%$ \\
\hline Contaminação $(5,0 \%)$ & $5,00 \%$ & $5,0 \times 0,75=3,75 \%$ \\
\hline
\end{tabular}

As moldagens dos corpos de prova seguiram um método de mistura que envolveu primeiramente os agregados (acompanhados da adição de pirita), que foram adicionados na betoneira e parte da água para a mistura destes e melhor homogeneização. Na sequência, foi adicionado o cimento e o restante da água com a betoneira já em movimento.

Os corpos de prova foram desformadas com 24 horas e encaminhados para cura submersa saturada com cal até completarem 28 dias de idade. Na sequência, as amostras foram sujeitas à uma condição de aceleração do mecanismo de degradação por ataque por sulfato interno, a qual consistiu na permanência delas dentro de um tanque com água constantemente aerada, visando a disponibilização constante de água e oxigênio para a ocorrência do ataque.

- Estudo em pasta:

Usando a mesma proporção dos aglomerantes usado no estudo em concreto, foram moldados corpos de prova de pasta em pequenos potes plásticos, de $20 \mathrm{ml}$, hermeticamente fechados, para o ensaio de porosidade. Foram consideradas 5 amostras para cada série, em cada idade, para análise estatística com o Teste de Tukey.

\subsection{Parâmetros avaliados}

\section{- Estudo em Concreto:}

A partir dos 28 dias de idade dos concretos, até os 1100 dias, foram realizados ensaios para obtenção de três parâmetros: densidade de corrente, potencial de circuito aberto e resistividade elétrica do concreto.

As leituras dos três parâmetros foram realizadas através do equipamento GECOR 10, o qual utiliza o eletrodo de referência de cobre/sulfato de cobre para análise do potencial de circuito aberto. Além disso, o equipamento usa a técnica de resistência a polarização linear para obtenção da densidade de corrente de corrosão e, para a resistividade elétrica do concreto, é empregada à técnica dos quatro eletrodos, método de Wenner.

Nenhum dos ensaios empregados possui referência normativa nacional para comparação dos valores obtidos com a probabilidade ou com a cinética de corrosão da estrutura de concreto. Assim sendo, os resultados foram analisados com base nos valores descritos na literatura. A Tabela 2 apresenta valores de referência, propostos por Andrade e Alonso (2004), que correlacionam a densidade de corrente de corrosão com o nível de corrosão.

Tabela 2: Critério de avaliação da densidade de corrente de corrosão do concreto.

\begin{tabular}{c|c}
\hline Densidade de corrente $\left(\mu \mathrm{A} / \mathrm{cm}^{2}\right)$ & Nível de corrosão \\
\hline$<0,1$ & Despresível \\
\hline $0,1-0,5$ & Baixo \\
\hline $0,5-1,0$ & Moderado \\
\hline$>1,0$ & Alto \\
\hline
\end{tabular}

Fonte: Andrade e Alonso (2004). 
Para a análise dos resultados do potencial de circuito aberto no concreto armado, foram utilizados os valores recomendados pela norma ASTM 876 (2015), apresetnados na Tabela 3. Cabe ressaltar que a ASTM 876 (2015) apresenta como informação que os intervalos propostos foram obtidos por meio de estudos experimentais de corrosão por ataque de cloretos, não sendo este o caso do presente estudo. Assim sendo, os valores foram utilizados como base para análise, mas levando em consideração possíveis mudanças nos intervalos pela diferença no ataque estudado.

Tabela 3: Critério de avaliação do potencial de circuito aberto no concreto armado

\begin{tabular}{c|c} 
Cobre/Sulfato de cobre & Probabilidade de Corrosão \\
\hline mais negativo que $-350 \mathrm{mV}$ & superior a $90 \%$ \\
\hline de -200 a $-350 \mathrm{mV}$ & incerta \\
\hline mais positivo que $-200 \mathrm{mV}$ & inferior a $10 \%$ \\
\hline
\end{tabular}

Fonte: ASTM C876 (2015).

Por último, os valores indicados pelo CEB (1991), apresentados na Tabela 4, foram utilizados como referência para a avaliação dos resultados de resistividade elétrica. No entanto, para a utilização dos intervalos propostos pelo CEB (1986) foi necessário analisar a necessidade ou não da aplicação de um fator forma para transformar a amostra em um meio semiinfinito UNE 83988-2 (2012). Considerando o ábaco apresentado por Gowers e Millard (1999), que leva em consideração a relação b/d (base do corpo de prova/distância equidistante entre os eixos dos eletrodos do resistivímetro) chegou-se em um fator de correção próximo a 1 para a amostra estudada.

Tabela 4. Critério de avaliação da resistividade elétrica do concreto.

\begin{tabular}{c|c}
\hline Resistividade Elétrica do Concreto $(\mathrm{k} \Omega . \mathrm{cm})$ & Indicação do risco de corrosão \\
\hline$>20$ & Despresível \\
\hline 10 a 20 & Baixa \\
\hline 5 a 10 & Alta \\
\hline$<5$ & Muito Alta \\
\hline
\end{tabular}

Fonte: CEB (1991).

\section{- Estudo em pasta:}

Nas idades determinadas $(28,84,168,360$ e 720 dias), as amostras foram recolhidas da condição em que estavam

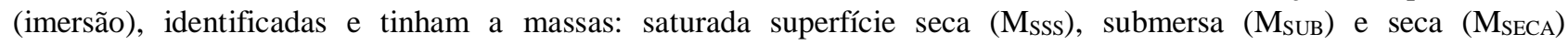
determinadas. Assim sendo, primeiramente, as amostras cilíndricas eram fragmentadas e cinco fragmentos eram coletados e secos superficialmente, para posterior determinação da massa saturada superfície seca (Msss). Na sequência, essas mesmas amostras eram pesadas em balança hidrostática para a determinação da massa submersa (Msub.). Após esta etapa, as amostras foram acondicionadas em recipiente polimérico e imergidas em álcool etílico p.a. durante 24 horas e, na sequência, retiradas do álcool e secas em estufa a $40{ }^{\circ} \mathrm{C}$ (24 horas). As amostras tiveram, então, a massa seca (MSECA) determinada em balança de precisão. A porosidade das pastas, nas idades de interesse, foi determinada por meio da Equação (1).

Porosidade $=\frac{\mathrm{M}_{\text {SSS }}-\mathrm{M}_{\text {SECA }}}{\mathrm{M}_{\text {SSS }}-\mathrm{M}_{\text {SUB }}} \cdot 100$

A média deste ensaio foi obtida por meio de 5 valores, 5 amostras para cada série. Os resultados obtidos neste ensaio foram tratados estatisticamente por meio do teste de Tukey, com $95 \%$ de confiança.

\section{RESULTADOS E DISCUSSÕES}

A Fig. 2 apresenta os resultados de densidade de corrente de corrosão e potencial de corrosão, em função do teor de contaminação por $\mathrm{SO}_{3}$ e das idades de envelhecimento. 


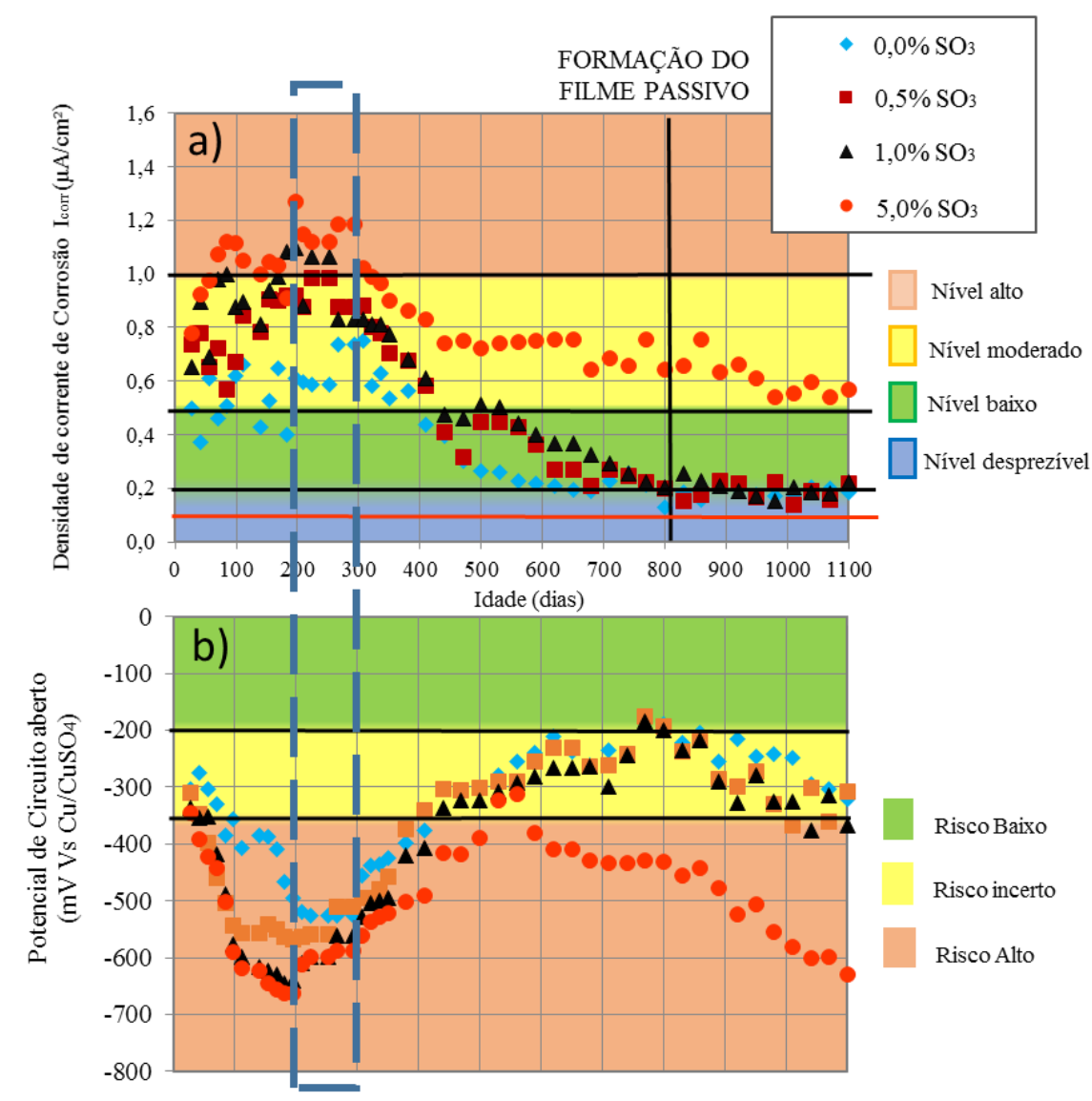

Figura 2: Monitoramento eletroquímico para as séries com e sem sulfetos: (A) densidade de corrente de corrosão; (B) potencial de circuito aberto.

Quanto a avaliação da densidade de corrente de corrosão [ $\mathrm{I}_{\text {corr }}$, de acordo com de acordo com Goni e Andrade (1990), existe uma zona de incerteza entre 0,1 (corrosão desprezível) e $0,2 \mu \mathrm{A} / \mathrm{cm}^{2}$ (corrosão ativa). Desse modo, Gonzalez et al. (1996) afirmam que apenas $I_{\text {corr }}$ maior do que a faixa de $0,1-0,2 \mu \mathrm{A} / \mathrm{cm}^{2}$ deve ser considerado como risco para a durabilidade do concreto armado. Sobre este tema, o trabalho de Andrade e Alonso (2004) reafirma que valores de $\mathrm{I}_{\text {corr }}$ abaixo de $0,1 \mu \mathrm{A} / \mathrm{cm}^{2}$ indica corrosão desprezível, de modo que a barra de reforço pode ser considerada como passiva. Porém, a faixa de valores entre 0,1 e $0,2 \mu \mathrm{A} / \mathrm{cm}^{2}$ pode ser considerada como uma zona de transição entre o estado passivo e o ativo de corrosão. Esta faixa de variação deve ser usada como limite de despassivação devido ao erro de fator 2 inerente a incerteza embutida na fórmula de Stern, de modo que uma leitura de $0,2 \mu \mathrm{A} / \mathrm{cm}^{2}$ pode ser perfeitamente igual a $0,1 \mu \mathrm{A} / \mathrm{cm}^{2}$. Desse modo, é comum outros autores considerarem que valores que atingem esta faixa de variação entrem em estado passivo, como os trabalhos de Poursaee e Hansson (2007) e o de Ortolna, Mancio e Tutikian (2016) que também investigaram o tempo de passivação das armaduras de aço carbono.

Nas idades iniciais, os resultados (Fig. 2a) indicam valores de densidade de corrente de corrosão acima de $0,5 \mu \mathrm{A} / \mathrm{cm}^{2}$, indicando corrosão ativa e moderada de acordo com a Tabela 3, para todas as séries. No entanto, há uma tendência clara de redução dos valores ao longo do tempo, até a estabilização dos dados. Para as séries com 0,0;0,5 e 1,0\% de $\mathrm{SO}_{3}$, os valores de $I_{\text {corr }}$ se estabilizam na faixa de $0,1-0,2 \mu \mathrm{A} / \mathrm{cm}^{2}$ após 800 dias de exposição. No entanto, a série com maior teor de contaminação $\left(5,0 \%\right.$ de $\left.\mathrm{SO}_{3}\right)$ apresentou valor de $\mathrm{I}_{\text {corr }}$ estabilizado acima de $0,5 \mu \mathrm{A} / \mathrm{cm}^{2}$, sendo a corrosão considerada corrosão moderada segundo a classificação indicada por Andrade e Alonso (2004), até os 1100 dias de idade. Este resultado indica que a série com o maior teor de contaminação tendeu a não passivar as armaduras de aço carbono do concreto armado.

Com relação ao potencial de circuito aberto (Fig. 2b), no início da análise altos valores negativos (-600mV/-700mV) foram observados para todas as séries. Este comportamento foi observado até 400 dias para todas as séries de ensaio. De acordo com Rocha (2012) e Medeiros et al. (2017), a tendência de os valores se tornarem mais positivos, após esse período, é explicada pela formação do filme passivo na superfície da armadura. 
Segundo Sun et al. (2017) e Williamson e Isgor (2016) o processo de formação do filme passivo não é totalmente compreendido, e muitos fatores podem interferir na formação da película protetora da armadura, como a concentração de íons hidroxila $\left(\mathrm{OH}^{-}\right)$nos poros e o uso de adições na mistura. A película de passivação é formada pelas reações eletroquímicas que ocorrem na interface armadura/concreto (Poursaee, 2016; Jiang et al., 2017 e Ribeiro et al., 2018). Godinho et al. (2019) observaram um período inicial de instabilidade nas leituras de Potencial de circuito aberto (Ecorr), possivelmente causado pelas reações de dissolução/precipitação inerente aos primeiros estágios da hidratação.

A formação da película de passivação atravessa três estágios: região ativa, região passiva e região de transição (Poursaee, 2016; Meira, 2017 e Ribeiro et al., 2018). Baseado nisso, no caso do monitoramento do potencial de circuito aberto e da densidade de corrente, pode-se identificar o trecho de corrosão ativa na faixa entre 200-300 dias. Antes disso, o potencial de circuito aberto apresenta tendência de se tornar cada vez mais eletronegativo e a densidade de corrente apresenta-se oscilando com valores entre o nível de corrosão moderado a alto, sendo caracterizado o fim da região ativa. Na sequência, inicia-se um trecho de redução dos valores de densidade de corrente e aumento de potencial de circuito aberto, sendo esta a região de transição, em que a película passivadora está em fase de formação, até chegar no estado de corrosão passiva (região passiva).

A Fig. 3 apresenta os resultados de resistividade elétrica para cada concreto estudado. Nota-se que o parâmetro avaliado apresentou tendência de crescimento ao longo do tempo. No Brasil não existe uma norma regulamentadora para o ensaio de resistividade elétrica superficial. Entretanto, os resultados podem ser avaliados usando os limites prescritos pelo boletim 192 do CEB (1991) mostrados na Tabela 4. O aumento desta propriedade ao longo do tempo, também observado por Polder (1995) e Medeiros-Junior et al. (2014), pode ser justificado pela constante formação dos produtos de hidratação do cimento Portland, que preenchem os poros, diminuindo os tamanhos e as ligações entre eles, dificultando a passagem de corrente e fazendo com que o valor de resistividade elétrica seja aumentado.

Nesse contexto, é importante destacar que a resistividade do concreto é uma propriedade de transporte do material, diretamente relacionada à rede de poros capilares dentro da matriz de cimento, que têm influência significativa na durabilidade e desempenho em longo prazo da estrutura de concreto armado (Medeiros, 2001; Mccarter et al., 2009). A Fig. 4 mostra a variação da porosidade total das amostras ao longo de 800 dias. Analisando a série de referência, fica claro que existe a redução da porosidade ao longo do tempo de hidratação do concreto. A mesma tendência existe para a série contaminada com $05 \%$ de pirita. Porém, no caso da contaminação com 1,0 e 5,0\%, o processo de ataque por sulfato interno proveniente da contaminação por pirita é suficiente para gerar microfissuras na matriz de cimento, aumentando a porosidade no ensaio de imersão em água.

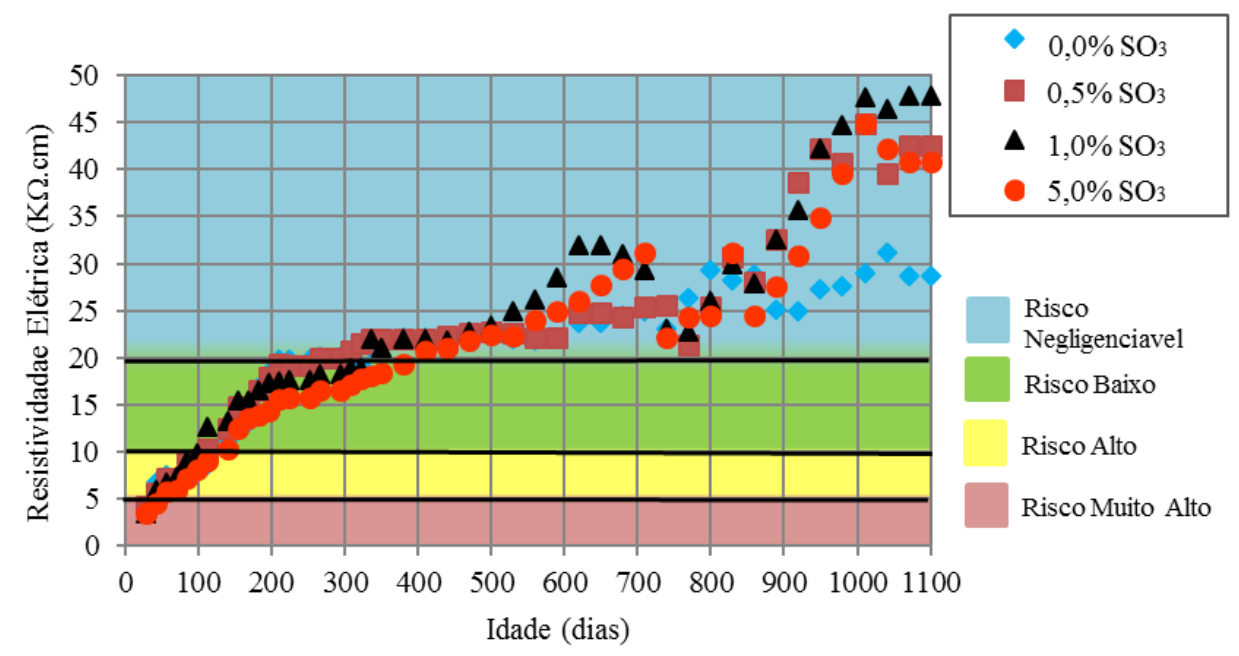

Figura 3: Monitoramento da resistividade elétrica das séries, com e sem sulfetos. 


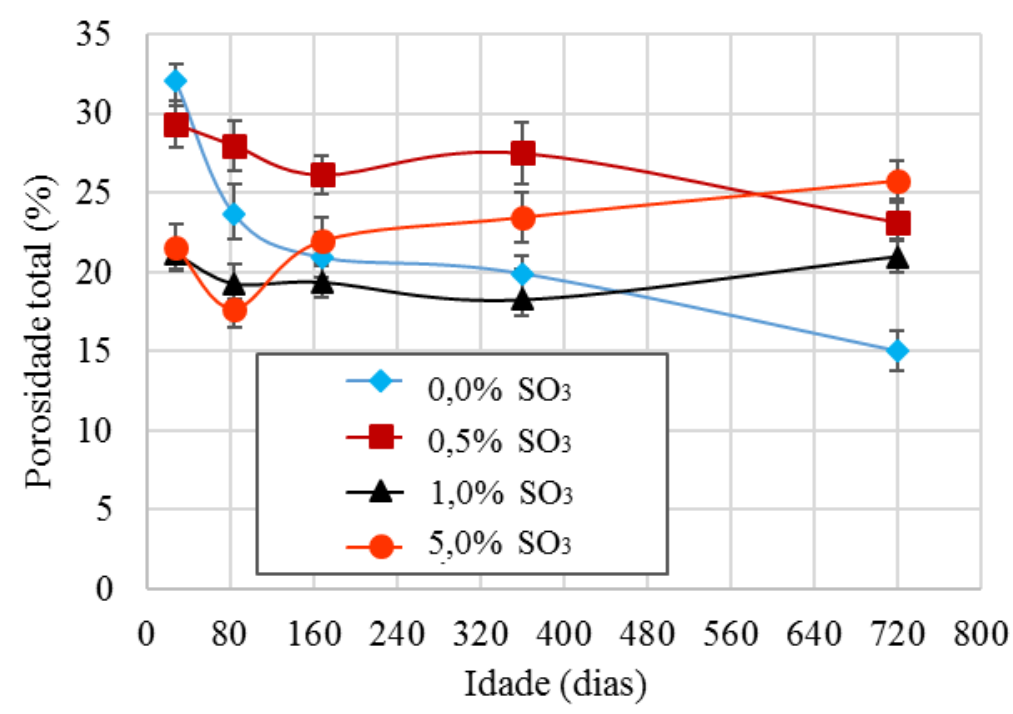

Figura 4: Porosidade das séries de ensaio, com e sem sulfetos.

\section{CONCLUSÕES}

Os dados experimentais possibilitam elaborar as conclusões a seguir:

- $\quad$ O potencial de circuito aberto e a densidade de corrente indicaram que a série com o maior teor de contaminação $\left(5,0 \%\right.$ de $\left.\mathrm{SO}_{3}\right)$ tem maior suscetibilidade à corrosão do aço no concreto. Para esta série não foi possível apontar a formação do filme passivo.

- $\quad$ Ficou comprovado que a passivação do aço embutido no concreto ocorre mesmo para os níveis de contaminação de $0,5 \%$ e $1,0 \%$ de $\mathrm{SO}_{3}$;

- $\quad$ O estudo demorou a evidenciar a formação de película passivadora nas barras de aço estudadas, podendo ser justificado pelo alto teor de pozolana, alta relação água/cimento e pela condição de exposição adotada. A passivação só pode ser identificada para as séries $0,0 \% \mathrm{SO}_{3}, 0,5 \% \mathrm{SO}_{3}$ e $1,0 \% \mathrm{SO}_{3}$;

- A resistividade elétrica foi um parâmetro crescente para as quatro séries durante o período estudado. Além disso os valores obtidos não destoaram entre os grupos, não sendo, então, o teor de contaminação fonte de variação nesta propriedade.

- Ao longo do tempo, a série de referência (sem contaminação por $\mathrm{SO}_{3}$ ) apresentou redução da porosidade total do concreto devido a evolução da hidratação.

- A porosidade aumentou para os níveis de contaminação de 1,0 e 5,0 \% de $\mathrm{SO}_{3}$ devido a expansão e consequente microfissuração da pasta.

\section{AGRADECIMENTOS}

Os autores agradecem o apoio financeiro fornecidos pela Coordenação de Aperfeiçoamento de Pessoal de Nível Superior (CAPES) e pelo Conselho Nacional de Desenvolvimento Científico e Tecnológico (CNPq). Também deve-se registrar o agradecimento pela disponibilização da infraestrutura do Centro de Estudos em Engenharia Civil (CESEC) da Universidade Federal do Paraná (CESEC/UFPR).

\section{REFERÊNCIAS}

ANDRADE, C.; MARTÍNEZ, I. Calibration by gravimetric losses o electrochemical corrosion rate measurement using modulated confinement of the current. Materials and Structures, v. 38, p. 833-841, 2005. 
ASSOCIAÇÃO BRASILEIRA DE NORMAS TÉCNICAS. ABNT:NBR NM 52: Agregado miúdo - Determinação da massa específica e massa específica aparente, 2009.

ASSOCIAÇÃO BRASILEIRA DE NORMAS TÉCNICAS. ABNT:NBR NM 53: Agregado graúdo - Determinação da massa específica, massa específica aparente e absorção de água, 2009.

AMERICAN CONCRETE INSTITUTE ACI 201: Guide to Durable Concrete. Detroit - USA, 1991.

ASSOCIATION FRANÇAISE DE NORMALISATION. AFNOR NF P 18-540: Granulats - Définitions, conformité, spécifications, 1997.

AMERICAN SOCIETY FOR TESTING AND MATERIALS. ASTM C876: Standard test method for corrosion potentials of uncoated reinforcement steel in concrete, 2015.

AMERICAN SOCIETY FOR TESTING AND MATERIALS. ASTM G-57: Standard test method for field measurement of soil resistivity using the Wenner. Four-electrode method, 2012.

ASOCIACIÓN ESPAÑOLA DE NORMALIZACIÓN Y CERTIFICACIÓN. UNE 83988-2: Durabilidad del hormigón - determinación de la resistividad - Parte 2: Método de las cuatro puntas o de Wenner, 2012.

ANDRADE, C.; ALONSO, C. Test methods for on-site corrosion rate measurement of steel reinforcement in concrete by means of the polarization resistance method. Materials and Structures, v. 37, p. 623-643, 2004.

ARAUJO, G. S. La reacción sulfática de origen interno en presas de hormigón. Propuesta metodológica de análisis. 2008. Tesis Doctoral. Universitat Politècnica de Catalunya. Departament d'Enginyeria de la Construcció. Barcelona, 2008.

ARAUJO D. ADRIANA; PANOSSIAN, ZEHBOUR; OLIV, KLEBER J.; FILHO PEREIRA, MARIO L. Técnicas de medição da resistividade elétrica em estruturas atmosféricas de concreto convencional. Revista téchnne. Edição 210 - Setembro, 2014.

BRANDÂO CAPRARO, ANA PAULA; VINICIUS BRAGA; MARCELO HENRIQUE FARIAS DE MEDEIROS; JUAREZ HOPPE FILHO; MARIANA O. G. P. BRAGANÇA; KLEBER FRANKE PORTELLA; ISABELA BLANCO, A.; F. PARDO-BOSCH; S. CAVALARO; A. AGUADO. Lessons learned about the diagnosis of pathologies in concrete dams: 30 years of research and practice. Construction and Building Materials, v. 197, p. 356-368, 2019.

CASANOVA, I.; AGULLÓ, L.; AGUADO, A. Aggregate expansivity due to sulfide oxidation - I. Reactions system and rate model. Cement and Concrete Research. v. 26, p. 993-998, 1996.

CRISTINA DE OLIVEIRA. Internal attack by sulphates in cement pastes and mortars dosed with different levels of pyrite. Journal of Building Pathology and Rehabilitation, v. 2. Julio, 2017.

COMITÉ EURO-INTERNACIONAL DU BÉTON. CEB: Diagnosis and assessment of concrete structures, 1989.

CENTURIONE, S. L.; KIHARA, Y.; BATTAGIN, A. F. Durabilidade de concreto submetido a ataques de íons sulfato. In: Congresso Brasileiro de Cerâmica, 47 ., 2003, João Pessoa. Anais [Proceedings of the 47th Annual Meeting of the Brazilian Ceramic Society], João Pessoa - PB - Brasil, 15-18/junho, 2003.

COUTINHO, J. S. Ataque por Sulfatos. Faculdade de Engenharia do Porto (FEUP), 2001.

CAMPOS, A.; C. M. LÓPEZ; A. BLANCO; A. AGUADO. Effects of an internal sulfate attack and an alkaliaggregate reaction in a concrete dam. Construction and Building Materials, v. 166, p. 668-683, 2018.

CAMPOS, A.; C.M.LÓPEZ; A. AGUADO. Diffusion-reaction model for the internal sulfate attack in concrete. Construction and Building Materials, v. 102, p. 531-540, 2016. 
CHUANBEI LIU; JIANMING GAO; FEI CHEN; YASONG ZHAO; XUEMEI CHEN; ZHIZHANG HE. Coupled effect of relative humidity and temperature on the degradation of cement mortars partially exposed to sulfate attack. Construction and Building Materials, v. 216, p. 93-100, 2019.

DODDS, WAYNE; GOODIER, CHRIS; CHRISTODOULOU, CHRISTIAN; AUSTIN, SIMON; DUNNE, DAVID. Durability performance of sustainable structural concrete: Effect of coarse crushed concrete aggregate on microstructure and water ingress. Construction and Building Materials, v. 145, p. 183-195, 2017.

FAQUINELI, C. R. Estudo da reatividade de agregados contendo sulfetos em concretos e argamassas. 2010. Dissertação apresentada a Universidade Federal de Minas Gerais, 2010.

G. GOMIDES, M. J. Investigação de agregados contendo sulfetos e seus efeitos sobre a durabilidade do concreto. Tese de doutorado. 2009. Programa de pós-graduação em engenharia civil. Universidade Federal do Rio Grande do Sul. Porto Alegre, 2009.

GOWERS, K. R.; MILLIARD, S. G. Measurement of concrete resistivity for assessment of corrosion severity of steel using Wenner technique. ACI Materials Journal, v. 96, p. 536-54, 1999.

GODINHO, JAYSON PEREIRA; ZERMIANI, NATALIA BAGGIO2; OLIVEIRA, RAFAEL LUIZ NEVES DE.; MEDEIROS, MARCELO HENRIQUE FARIAS DE. Passivação do aço carbono nas idades iniciais de hidratação do concreto armado. In: Simmposio Paranaense de Patologia das Construcoes (SPPC), 4, 2019, Curitiba - PR. Anais. ISSN 2526-7248. Artigo n. 4SPPC129, p. 271-281, 2019.

GONI, S.; ANDRADE, C. Synthetic concrete pore solution chemistry and rebar corrosion rate in the presence of chlorides. Cement and Concrete Research, v. 20, p. 525-539, 1990.

HELENE, P. R. L. Contribuição ao estudo da corrosão em armaduras de concreto armado. 1993. Tese de doutorado apresentada à Escola Politécnica da Universidade de São Paulo, 1993.

HASAN ŞAHAN AREL; BLESSEN SKARIAH THOMAS. The effects of nano- and micro-particle additives on the durability and mechanical properties of mortars exposed to internal and external sulfate attacks. Results in Physics, v. 7, p. 843-851, 2017.

HOSSACK, ASHLEE M.; THOMAS MICHAEL D.A. The effect of temperature on the rate of sulfate attack of Portland cement blended mortars in Na2SO4 solution. Cement and Concrete Research, v. 73, p. 136-142., 2015.

HASPARYK NP, MUNIZ FC, ANDRADE MAS, GOMIDES MJ, BITTENCOURT RM, CARASEK H. The deterioration of concrete structures containing aggregates with sulphides. In: Epusp symposium on concrete structures. Anais, 2003.

IKUMI, TAI; SEGURA, IGNACIO; CAVALARO, SERGIO H.P. Influence of early sulfate exposure on the pore network development of mortars. Construction and Building Materials, v. 143, p. 33-47., 2017.

IKUMI, TAI; SEGURA, IGNACIO; CAVALARO, SERGIO H.P. Alternative methodology to consider damage and expansions in external sulfate attack modeling. Cement and Concrete Research, v. 63, p. 105-116., 2014.

LMAN OLIVEIRA; SERGIO H.P. CAVALARO; ANTONIO AGUADO. New kinetic model to quantify the internal sulfate attack in concrete. Cement and Concrete Research, v. 43, p. 95-104., 2013.

JIANG, J.Y.; WANG, D.; CHU, H.Y.; MA, H.; LIU, Y.; GAO, Y.; SHI, J.; SUN, W. The passive film growth mechanism of new corrosion-resistant steel rebar in simulated concrete pore solution: Nanometer structure and electrochemical study. Materials (Basel), v. 10, p. 412, 2017.

KOGA, G. Y.; ALBERT, B.; ROCHE, V.; NOGUEIRA, R. P. A comparative study of mild steel passivation embedded. In Belite-Ye'elimite-Ferrite and Portland cement mortars. Electrochimica Acta, v. 261, p. 66-77, 2018.

LINO MAIA; SERGIO ALVES. Low durability of concrete elements due to steel corrosion - cases wherein the steel reinforcing bars acted as an internal clock bomb. In: Internacional Conference on Structural Integrity (ICSI). 2nd, 4 - 7 September, 2017, Funchal, Madeira, Portugal. Procedia Structural Integrity, v. 5, p. 139-146, 2017. 
LI D, ZHANG S, YANG W, ZHANG W. Corrosion monitoring and evaluation of reinforced concrete structures utilizing the ultrasonic guided wave technique. Int J Distrib Sens Netw, v. 827130, p. 1- 9, 2014.

MEIRA, G.R. Corrosão de Armaduras em Estruturas de Concreto - fundamentos, diagnóstico e prevenção. In: 1a edição do IFPB, 1, 2017, João Pessoa - PB. Anais, 2017.

MEHTA, P.K.; MONTEIRO, P.J. Concreto: Microestrutura, Propriedades e Materiais. In: IBRACON, 2a edição, 2014, São Paulo, Brasil, Anais, 2014.

MEDEIROS, M. H. F. Estudo de variáveis que influenciam nas medidas de resistividade de estruturas de concreto armado. Teses. 2001. Escola Politecnica da Universidade de São Paulo - SP, Brasil, 2001.

MEDEIROS, M. H. F.; ROCHA, F. C.; MEDEIROS JUNIOR, R. A.; HELENE, P. Corrosion potential: influence of moisture, water-cement ratio, chloride content and concrete cover. Revista IBRACON de estruturas e materiais, v. 10, p. 864-885., 2017.

MCCARTER, W. JOHN; STARRS, GERRY; KANDASAMI, SIVA; JONES, ROD AND CHRISP, MALCOLM. Electrode Configurations for Resistivity Measurements on Concrete. ACI Materials Journal, v. 106-M30, 2009.

MEDEIROS-JUNIOR, R. A.; LIMA, M. G.; MEDEIROS, M. H. F.; REAL, L. V. Investigação da resistência à compressão e da resistividade elétrica de concretos com diferentes tipos de cimento. Alconpat, v. 4, p. 116-128, 2014.

MEDEIROS, M. H. F.; ANDRADE, J. J. O.; HELENE, P. Durabilidade e vida útil das estruturas de concreto. Concreto: Ciência e Tecnologia - IBRACON, 2011.

OKENIYI JO; LOTO CA; POPOOLA API. Rhizophora mangle L. Effects on steel-reinforced concrete in 0.5 M H2SO4: Implications for corrosion degradation of wind-energy structures in industrial environments. Energy Procedia, v. 50, p. 429-436, 2014.

OLIVEIRA, I. Reacción sulfáticas interna en presas de hormigón: cinética del comportamiento. Tesis Doctoral. 2011. Universitat Politècnica de Catalunya. Departament d’Enginyeria de la Construcció. Barcelona, 2011.

PARANDE AK, RAMSAMY PL, ETHIRAJAN S, RAO CRK, PALANISAMY N. Deterioration of reinforced concrete in sewer environments. Proc. Inst Civ Eng Munic Eng, v. 159, p. 11-20, 2006.

POURSAEE A. Corrosion of Steel in Concrete Structures. In: Elsevier, 1st Edition. 2016, Amsterdã, Holanda. Anais, 2016.

POLDER, R. B. Chloride diffusion and resistivity testing of five concrete mixes for marine environment. In: 1st RILEM workshop on Chloride Penetration into Concrete, 1, 1995. Anais, p. 9, 1995.

PRUCKNER, F. Corrosion and protection of reinforcement in concrete measurements as interpretation. Thesis of Faculty of Natural Sciences and Mathematics, 2001.

PEREIRA, E.; BRAGANÇA, M. O. G. P.; OLIVEIRA, I. C.; GODKE, B.; PORTELLA, K. F. Ataque interno por sulfatos em estruturas de concreto contaminadas por pirita: Uma revisão da literatura. In: $1^{\circ}$ Congresso Brasileiro de Patologias das Construções. 1, 21 a 23 de maio de 2014, Foz do Iguaçu, Paraná. Anais, 2014.

ROCHA, F, C. Leituras de potencial de corrosão em estruturas de concreto armado: influência da relação água/cimento, da temperatura, da contaminação por cloretos, da espessura de cobrimento e do teor de umidade do concreto. Dissertação de mestrado apresentada à Universidade Federal do Paraná, 2012.

Ribeiro, D. V.; Helene, P. ; CASCUdO, O. ; TUtiKian, B. F. ; SAlES, A. ; SOUZA, C. A. C. ; CUNHA, M. P. T.; LOURENCO, M. Z.; ALMEIDA, F. C. R. Corrosão e degradação em estruturas de concreto: Teoria, controle e técnicas de análise e intervenção. In: Elsevier, 2a Edition, Elsevier Brasil, Rio de Janeiro-RJ. Anais, 2018. 
SUN, X. H.; ZUO, X. B.; YIN, G. J.; JIANG, K.; TANG, Y. J. Electrochemical and microscopic investigation on passive behavior of ductile iron in simulated cement-mortar pore solution. Construction and Building Materials, $\mathrm{v}$. 150, p. 703-713, 2017.

SANTOS T.M.H., Corrosão das Armaduras do Betão Armado Causas, Consequências, Prevenção e Projeto de Durabilidade. In: IST (MSc). Lisbon. Anais, 2014

WILLIAMSON, J.; ISGOR, O. B. The effect of simulated concrete pore solution composition and chlorides on the electronic properties of passive films on carbon steel bar. Corrosion Science, v. 106, p. 82-95, 2016.

ZHUO TANG; WENGUI LI; GUOJUN KE; JOHN L. ZHOU; VIVIAN W.Y.TAM.M. Sulfate attack resistance of sustainable concrete incorporating various industrial solid wastes. Journal of Cleaner Production, v. 218, p. 810-82., 2019.

ZANQUN LIU; WENLONG HU; LE HOU; DEHUA DENG. Effect of carbonation on physical sulfate attack on concrete by $\mathrm{Na}_{2} \mathrm{SO}_{4}$. Construction and Building Materials, v. 193, p. 211- 220., 2018. 